\title{
3D VELOCITY ANALYSIS USING LIMITED APERTURE MIGRATION
}

\section{Summary}

Migration velocities can be determined by performing several prestack migrations with a series of test velocity models. The velocity model that produces a consistent image for all source-receiver offsets is taken to be the correct migration velocity.

Limited-aperture migration involves migrating with aperture widths on the order of a Fresnel zone. Incorporating limited-aperture migration in prestack migration velocity analysis yields the following benefits:

- About a 10 fold reduction in computer time for 2D migration velocity analysis,

- About a 100 fold reduction in computer time for 3D migration velocity analysis,

- Higher signal-to-noise ratio for velocity analysis displays,

- Elimination of one type of false velocity event from velocity sweep displays.

\section{Introduction}

Many methods for determining migration velocities involve prestack migrating with a number of test velocity models (Al-Yahya 1989; Deregowski 1990; Schleicher, Grygier et al. 1991). The velocity model which produces the most consistent images for all source receiver offsets is taken to be the correct migration velocity. These velocity analysis methods, which involve several applications of prestack migration, can be prohibitively expensive when applied to 3D data. The cost of 3D prestack migration must be reduced to make these methods widely applicable.

One way of reducing the cost of prestack migration is to use an inherently fast migration technique. There are three commonly used wave-equation migration algorithms: frequency-wavenumber, finite-difference, and Kirchhoff. Frequency-wavenumber and finite-difference migrations are generally faster than Kirchhoff migration; however, the following advantages of Kirchhoff migration have made it the method of choice for 3D prestack migration:

1. Can handle irregular shooting geometries,

2. Can use complex migration velocity fields,

3. Can operate in a target-oriented mode,

4. Can accurately migrate reflectors having very steep dip.

Frequency-wavenumber migration fails under conditions 1,2 and 3 above, while finite-difference migration fails under conditions 1,3 and 4 . Therefore, I will only consider methods for speeding up Kirchhoff migration.

The equations describing Kirchhoff migration are well known (Berryhill 1984; Schneider 1978). Kirchhoff migration involves summing the input seismic traces along traveltimes corresponding to a point diffractor in the subsurface. The migration-aperture is defined as all the traces we include in this summation for a given output trace. The aperture is usually on the order of 100 Fresnel zones around the output trace (usually about 1.5 to 10 $\mathrm{km})$.

\section{Method}

For reflections, only about one Fresnel zone of traces within the migration aperture contribute significantly to the output image. Those input traces that contribute significantly can be determined from the reflection time dip by using ray tracing. Including only those traces that contribute significantly in the Kirchhoff summation speeds up 2D 
prestack migration by about a factor of 10 and speeds up 3D prestack migration by about a factor of 100 .

Conventionally, an output image is formed along a vertical line at the center of the migration aperture. However, when migrating small apertures, reflections with even a small amount of dip will migrate out of the region defining the aperture. Two methods can be used to solve this problem. One method, called the fixed output location method, is to sum only those traces that contribute significantly to reflectors at a fixed output location (Carroll, Hubbard et al. 1987). In this case, the input trace aperture will vary with time along the output trace (see Fig. 1). A second method for limited-aperture migration, called the fixed input aperture method, is to select a small fixed aperture of input traces and compute migrated traces at the locations where reflections within the small aperture will migrate (Krebs 1990). In this mode, the lateral location of the output trace will vary with time (see Fig. 2).

The fixed input aperture method is computationally more efficient than the fixed output location method, because fewer input traces are needed to construct an output migrated trace (see Figs. 1 and 2). This results in less I/O for the fixed input aperture method relative to the fixed output location method. An added benefit of the fixed input aperture method is that it eliminates false velocity events from velocity sweep displays that are caused by discontinuous reflections migrating into and out of the velocity analysis location (Schleicher, Grygier et al. 1991).

In order to gain a computational advantage from limited-aperture migration we must have an inexpensive method for determining the output image curves. There are many methods for defining these curves. One good method is to determine the time dips of reflections at the center of the limited aperture to be migrated. Kinematic migration (often called map migration (Maher and Hadley 1985)) is used to predict, from these time dips, where the reflections will migrate. This kinematic migration is performed with the same reference migration velocity that will be used for prestack migration velocity analysis. Output image curves are then defined which pass through the output locations defined by kinematic migration (see Fig. 2).

Limited-aperture migration produces migrated seismic data with higher $\mathrm{S} / \mathrm{N}$ than conventional migration (Carroll, Hubbard et al. 1987; Krebs 1990). The reason for this is that limited-aperture migration only sums over those portions of the Kirchhoff summation curve that make a significant contribution to the migration of the reflection of interest. The remaining portions of the summation curve usually do not sum to zero. This non-zero sum is noise that conventional wide-aperture migration adds to the image of the reflection.

\section{Example}

The following is an example of limited-aperture migration velocity analysis using common-offset migration as discussed in the previous section. The data are from a 3D marine survey. The data were processed as shown in Fig. 3. This method converged after four iterations of prestack common-offset migration. Other methods of quantifying moveout and updating the velocity could be substituted, and may lead to faster convergence.

The velocity analysis was performed using conventional wide-aperture prestack migration (aperture $8,000 \times 2,400$ meters) and limited-aperture migration (aperture 1,333×267 meters). Both migrations yielded the same velocity function. This confirms the accuracy of the limited-aperture migration velocity analysis.

Figure 4 compares migrated CDP gathers resulting from common offset prestack migration processes shown in Figure 3. Migrated CDP gathers resulting from wide-aperture and limited-aperture $3 \mathrm{D}$ prestack migration are shown. The accuracy of the limited-aperture migration is demonstrated by the fact that the reflections in these CDP gathers have the same arrival times as a function of offset. Also, notice the increased $\mathrm{S} / \mathrm{N}$ of the limited-aperture migration relative to the conventional wide-aperture migration.

The CPU time for the limited-aperture migration was 55 seconds on a single CPU Cray-YMP. The time for the conventional wide-aperture migration was 2,756 seconds. Thus the limited-aperture migration was about 50 times faster than the conventional method.

\section{Conclusions}

Limited-aperture migration velocity analysis has the following advantages:

1. Reduces CPU time for migration velocity analysis.

2. Increases $\mathrm{S} / \mathrm{N}$ of velocity analysis displays.

3. Eliminates false velocity events from migration velocity sweep displays.

The examples discussed here all involve using limited-aperture migration to determine rms average velocities. Such velocities will not be satisfactory in areas requiring depth migration, since they do not account for raypath bending. The limited-aperture migration method can be extended to include raypath bending by using raytracing to determine the traveltimes required by the Kirchhoff integral. However, for depth migration velocity analysis, very fast raytracing methods will be required so that the cost of raytracing will not dwarf any savings realized by using 
limited-aperture migration. Furthermore, limited-aperture migration requires knowledge of reflector dips, which can usually be obtained from stacked seismic data. However, when raypath bending is significant, stacked seismic data may not be of sufficient quality to accurately measure reflection dips. In such cases, another source of dip information must be found or limited-aperture migration velocity analysis cannot be used.

\section{References}

Al-Yahya, K. M. (1989). “Velocity analysis by iterative profile migration.” Geophysics 54(6): 718-729.

Berryhill, J. R., Ed. (1984). Wave equation datuming before stack. 54th Annual Internat. Mtg. Soc. Expl. Geophys. Expanded Abstracts. Tulsa, Soc. Expl. Geophys., Session:S2.6.

Carroll, R. J., L. M. Hubbard, et al. (1987). A directed-aperture Kirchhoff migration. Geophysical imaging. symposium of Geophysical Society of Tulsa. Tulsa, Soc. Expl. Geophys. 151-165.

Deregowski, S. M. (1990). “Common-offset migrations and velocity analysis.” First Break 8(6): 224-234.

Krebs, J. R. (1990). “Three-dimensional migration of swath surveys.” Geophysics 55(9): 1251-1259.

Maher, S. M. and D. M. Hadley, Ed. (1985). Development of an accurate, stable, and interactive map migration algorithm. 55th Annual Internat. Mtg., Soc. Expl. Geophys., Expanded Abstracts. Tulsa, Soc. Expl. Geophys., Session:S15.8.

Schleicher, K. L., D. J. Grygier, et al., Ed. (1991). Migration velocity analysis: A comparison of two approaches. 61st Annual Internat. Mtg.. Soc. Expl. Geophys. Expanded Abstracts. Tulsa, Soc. Expl. Geophys., 1237-1238.

Schneider, W. A. (1978). "Integral formulation for migration in two-dimensions and three-dimensions." Geophysics 43(1): 49-76.

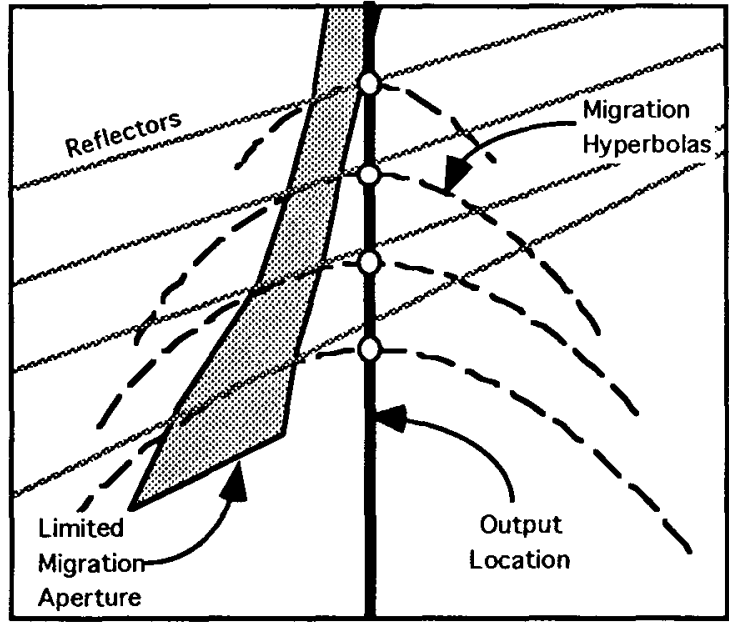

Figure 1 - Schematic diagram illustrating the fixed output location limited-aperture migration method.

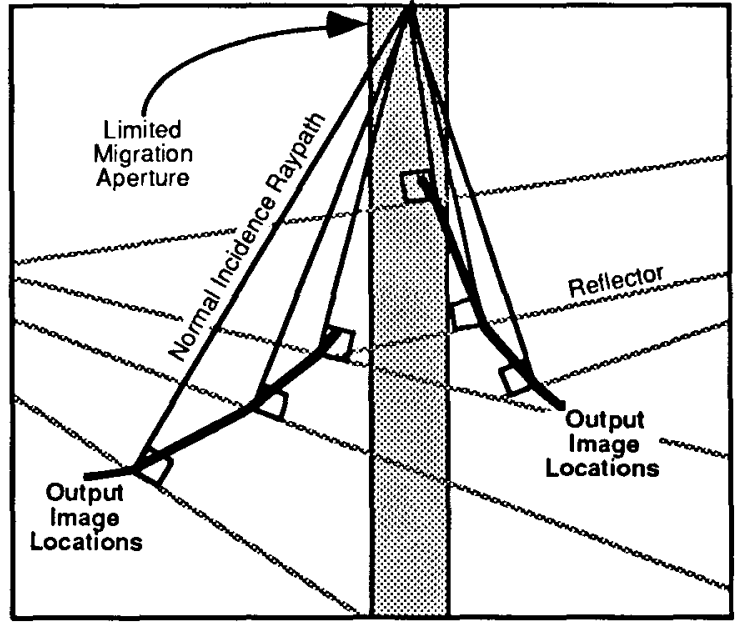

Figure 2 - Schematic diagram illustrating the fixed input aperture limited-aperture migration method. 


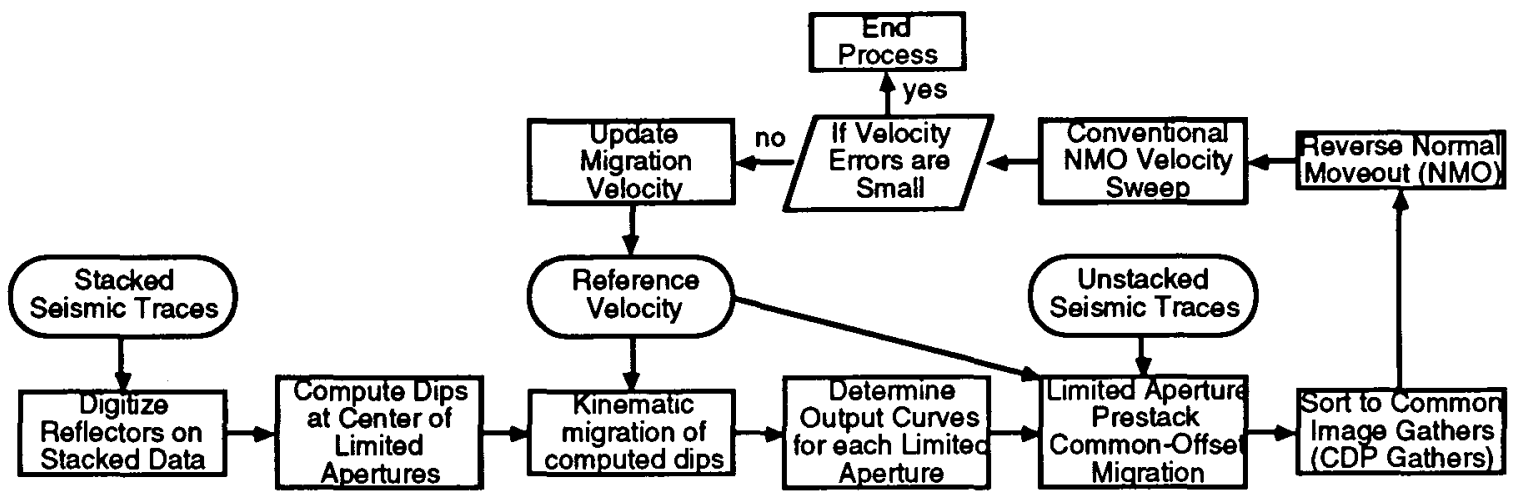

Figure 3 - Processes used to for limited-aperture migration velocity analysis.

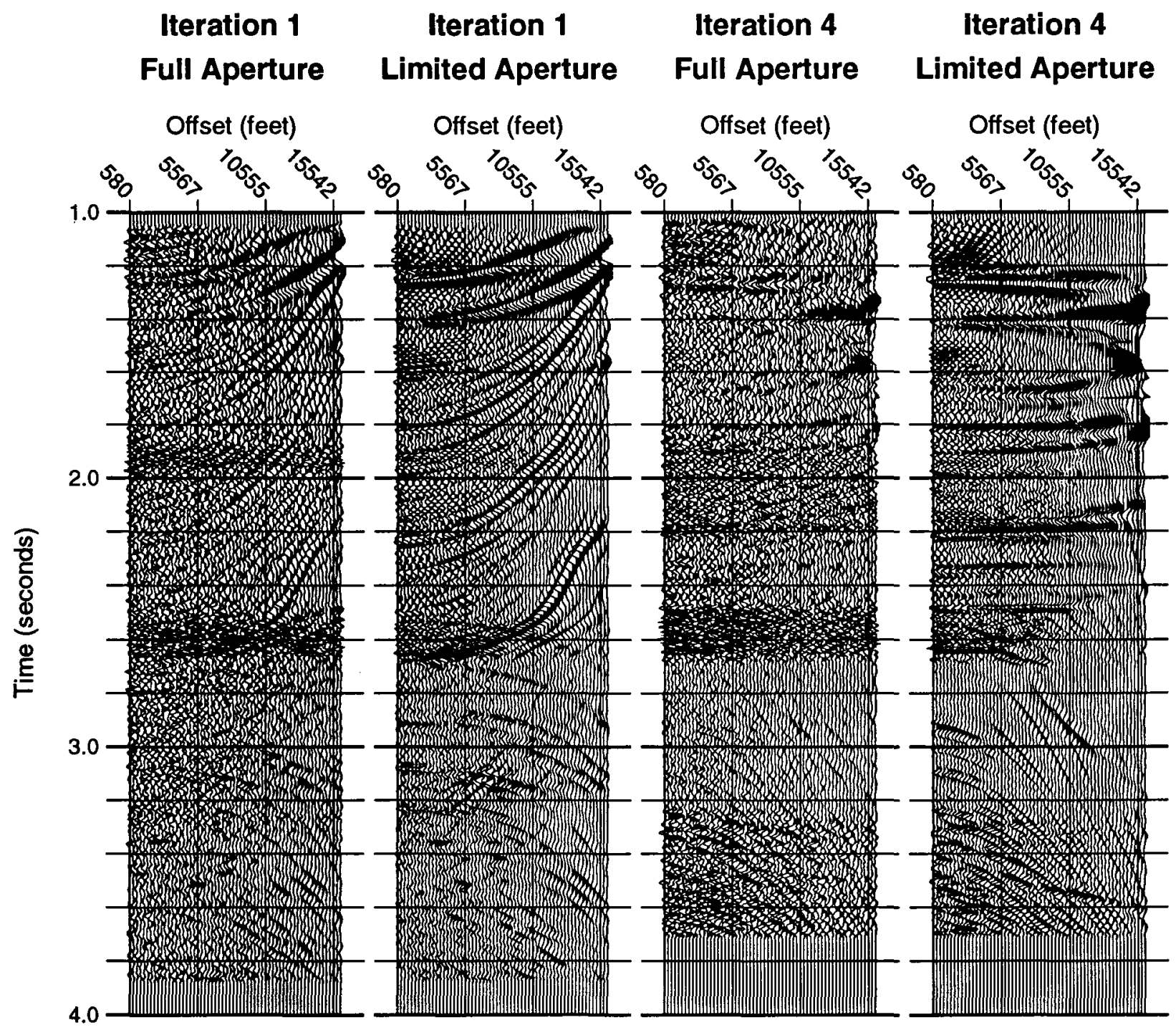

Figure 4 - Migrated CDP gathers comparing full-aperture and limited-aperture common-offset migration. Migrated gathers from iteration 1 using an initial guess at the reference velocity, and gathers following 4 iterations of the velocity analysis procedure are shown. 